\title{
Perioperative immunotherapy for pancreatic cancer is on its way
}

\author{
Christoph Springfeld ${ }^{1}$, Peter Bailey ${ }^{2,3}$, Thilo Hackert ${ }^{2}$, John P. Neoptolemos ${ }^{2}$ \\ ${ }^{1}$ Department of Medical Oncology, National Center for Tumor Diseases, Heidelberg University Hospital, Heidelberg, Germany; ${ }^{2}$ Department of \\ Surgery, Heidelberg University Hospital, Heidelberg, Germany; ${ }^{3}$ Institute of Cancer Sciences, University of Glasgow, Scotland, UK \\ Correspondence to: Prof. Dr. med. John P. Neoptolemos, MA, MB, BChir, MD, FRCS, FMedSci, MAE. Professor of Surgery, Department of General, \\ Visceral and Transplantation Surgery, University of Heidelberg, Im Neuenheimer Feld 420, 69120 Heidelberg, Baden-Württemberg, Germany. \\ Email: John.Neoptolemos@med.uni-heidelberg.de. \\ Comment on: Hewitt DB, Nissen N, Hatoum H, et al. A phase 3 randomized clinical trial of chemotherapy with or without algenpantucel-L \\ (hyperacute-pancreas) immunotherapy in subjects with borderline resectable or locally advanced unresectable pancreatic cancer. Ann Surg 2020. doi: \\ 10.1097/SLA.0000000000004669. [Epub ahead of print].
}

Submitted Jun 11, 2021. Accepted for publication Jun 28, 2021.

doi: $10.21037 / \mathrm{hbsn}-21-238$

View this article at: https://dx.doi.org/10.21037/hbsn-21-238

Interdisciplinary perioperative treatment has greatly improved the outcome of pancreatic cancer patients. Based on randomized, controlled phase III clinical trials, adjuvant chemotherapy with mFOLFIRINOX (PRODIGE-24 study), gemcitabine/capecitabine (ESPAC-4) or 5-fluorouracil/ gemcitabine monotherapy (ESPAC-1, CONKO-001, ESPAC-3) is now the clinical standard after curative resection (1). The only randomized controlled trial in borderline resectable patients comparing neoadjuvant therapy with upfront surgery is the phase II ESPAC$5 \mathrm{~F}$ feasibility study (2). Patients were randomized into four groups, the first three with neoadjuvant therapy: (I) FOLFIRINOX, (II) gemcitabine with capecitabine, and (III) chemoradiotherapy, versus (IV) upfront surgery (2). Neoadjuvant therapy was associated with a significant better overall survival than immediate surgery (2). In patients with locally advanced, unresectable pancreatic cancer, a small randomized trial showed that overall survival with attempted upfront surgery was significantly superior compared to chemoradiotherapy only without attempted resection (3). The evidence for using neoadjuvant therapy for unresectable pancreatic cancer to improve resection rates and/or survival is of low quality as no randomized studies have ever been undertaken. The largest single center cohort series from Heidelberg Germany provides some evidence that induction chemotherapy using FOLFIRINOX can provide better survival in those in whom conversion to resection is possible compared to those in whom resection is not possible (4). Gemcitabine-based regimens with nab-paclitaxel or capecitabine are reasonable alternatives FOLFIRINOX, with less toxicity based on the NEOLAP-AIO-PAK-0113 (NCT02125136) and ESPAC-5F trials (3).

Although sometimes used, the evidence for perioperative radiotherapy to improve survival is very limited. There are no randomized phase III data supporting the use of adjuvant radiochemotherapy after resection. In patients with locally advanced pancreatic cancer, the LAP-007 study also failed to show a survival benefit for the addition of radiotherapy in patients who did not progress after four months on chemotherapy. In the ESPAC-5F trial, neoadjuvant therapy with either FOLFIRINOX, or gemcitabine with capecitabine had much better survival than neoadjuvant chemoradiotherapy, whilst in the recent Alliance A021501 randomized phase II study with borderline resectable patients, neoadjuvant mFOLFIRINOX plus radiotherapy also resulted in an inferior outcome compared to mFOLFIRINOX alone $(3,5)$.

Immunotherapy has revolutionized the treatment of many types of cancer and is now also increasingly used in the perioperative setting. Adjuvant immunotherapy with checkpoint inhibitors after melanoma resection is recommended by current guidelines. It is therefore important to answer the question whether immunotherapy should also be included as a component of perioperative management of pancreatic cancer.

Hewitt et al., now report the results of the randomized phase III PILLAR study that tested the addition of algenpantucel-L to radiochemotherapy in patients with 
borderline resectable or unresectable, locally advanced pancreatic cancer (6). Algenpantucel-L is an intradermal vaccine made of two irradiated pancreatic cancer cell lines genetically modified to express murine $\alpha 1,3-$ galactosyltransferase. The mouse protein is recognized by human antibodies, causing hyperacute rejection of the transgenic cells. This immune response is expected to extend to the vaccinee's cancer cells by epitope spreading (7). Following a one-arm phase II study of 70 patients that received adjuvant chemotherapy and radiochemotherapy in combination with algenpantucel-L, the randomized phase III IMPRESS study with 722 patients was conducted, but was negative for the primary endpoint of overall survival (NewLink Genetics May 9, 2016). In the PILLAR study, 303 patients with borderline resectable $(n=53)$ or locally advanced pancreatic cancer $(n=250)$ were treated with chemotherapy followed by chemoradiation and randomized to either receive concomitant immunotherapy with Algenpantucel-L or not (6). The induction chemotherapy protocol was up to the investigator; about two-thirds of the patients received FOLFIRINOX, the others gemcitabine/ nab-paclitaxel. Patients with at least stable disease after chemotherapy were consecutively treated with external beam radiation (50.4 Gy in 28 fractions) with infusional 5 -fluorouracil or capecitabine. The primary endpoint of overall survival, was also negative. Survival was longer in the standard group (13.4 months) than in the immunotherapy group (12.4 months), the difference was not statistically significant. Subsequent resection was possible in $24 \%$ of patients. Resected patients had an overall survival of 28.7 months compared to 12.6 months in unresected patients. Vaccination did not improve survival in either of the subgroups (6).

One of the potential problems with the assessment of tumor responses to various therapeutic strategies is the concomitant use of radiotherapy, despite lacking randomized evidence to support its application for overall survival. Seifert et al., have shown in an experimental system comprising genetically engineered KPC mice, mimicking human pancreatic cancer, and C57BL/6 mice with orthotopic KPC tumors, that radiation treatment promoted advanced pancreatic intraepithelial lesions and more foci of invasive cancer than unexposed mice, with a reduced survival time of $>6$ months (8). Neoplastic lesions following radiation had an immune-suppressive macrophage 2-like phenotype with fewer $\mathrm{CD} 8^{+} \mathrm{T}$ effector cells, and more $\mathrm{CD}^{+}{ }^{+} \mathrm{T}$-cells of T-helper 2 and T-regulatory cell phenotypes and increased secretion of macrophage colony- stimulating factor 1 (MCSF-1). Moreover, the adoptive transfer of T-cells from irradiated tumors to the orthotopic $\mathrm{KPC}$ tumors in the C57BL/6 mice accelerated tumor growth but was slowed by using neutralizing antibody to MCSF-1, associated with alteration of the tumor macrophage phenotype, and increased anti-tumor T-cell response (8).

Pancreatic cancer has been considered to be an immunologically cold tumor microenvironment, defined by a dominant myeloid cell infiltration, devoid of $\mathrm{CD}^{+} \mathrm{T}$-cells, and low activation markers reflecting dysfunctional adaptive T-cell immunity and checkpoint blockade refractoriness (9). Oncogenic $m K R A S$ is present in $90 \%$ or more of pancreatic cancers, and in addition to it its direct oncogenic actions, it also promotes immunosuppression by multiple mechanisms (9). Bailey et al. have described four pancreas cancer subtypes based on differential transcription factor expression and downstream targets responsible for lineage specification and differentiation during development and regeneration: (I) squamous, (II) pancreatic progenitor, (III) immunogenic, and (VI) aberrantly differentiated endocrine exocrine (ADEX) (11). The immunogenic subtype is associated with immune gene programmes involving B-cell signaling pathways, antigen presentation, $\mathrm{CD} 4^{+} \mathrm{T}$-cell, $\mathrm{CD}^{+} \mathrm{T}$-cell and Toll-like receptor signaling pathways (10). Upregulation of programmed cell death protein (PD-1), and cytotoxic T-lymphocyte-associated protein 4 (CTLA-4) acquired tumor immune suppression pathways in this immunogenic subtype may offer therapeutic opportunities (10). Current immune-modulatory strategies must be multimodal aiming to (I) enhance endogenous T-cell function, (II) adoptively transfer tumor-specific T-cell immunity, and (III) attempt to devise an immunologically hot tumor microenvironment (9).

Balachandran et al. found that patients with pancreatic cancer having both the highest tumor neoantigen number (notably to the tumor antigen MUC16/CA125) and the most abundant $\mathrm{CD}^{+} \mathrm{T}$-cell infiltrates, had improved survival (11). Long-term survivors had neoantigens with differential presentation and homology to infectious disease-derived peptides with predicted cross-reactive microbial epitopes (11). Moral et al., have recently shown that group 2 innate lymphoid cells (ILC2s) infiltrate pancreatic ductal adenocarcinomas to activate tissue-specific tumor immunity, inferring another novel immunoregulatory target (12). These tumor infiltrating ILC2s (which express the PD-1 receptor) were enriched in long-term survivors with an immunologically hot tumor microenvironment 
containing abundant activated $\mathrm{CD} 8^{+} \mathrm{T}$-cells, and containing higher bulk tumor RNA expression of the ILC2-activating cytokine IL33 (12). Experimentally, it was shown that PD-1 blockade released ILC2 cell-intrinsic inhibition to expand and activate the tumor ILC2s to release CCL5 resulting in $\mathrm{CD}_{103^{+}}$dendritic cell expansion and then $\mathrm{CD} 8^{+} \mathrm{T}$-cell activation leading to enhanced anti-tumor immunity (12).

Targeted ablation of myeloid cells in PDAC, alone or in combination with immune checkpoint inhibitors, represents a promising alternative to existing immunotherapeutic approaches. Pre-clinical studies in KPC mice models have suggested that specific targeting of macrophages and neutrophils using small molecule inhibitors, specific for either the macrophage receptor CSF1R or the neutrophil receptor CXCR2, might facilitate better outcomes by enhancing endogenous T-cell cancer killing functions and the reprogramming of tumour cell intrinsic phenotypes $(13,14)$.

The FDA has given approval to pembrolizumab (an antibody for PD-1) for the treatment of microsatellite instability-high solid tumors, although this is present in only $1-3 \%$ of pancreatic cancers. Inhibition of the CXCR4CXCL12 pathway in pancreatic cancer also enhances tumor sensitivity to anti-PD-1 ligand-1 treatment. In the two-cohort phase IIa, COMBAT study (NCT02826486) pembrolizumab was combined with BL-8040 (a CXCR4 antagonist) in metastatic pancreatic cancer (15). The disease control rate was $34.5 \%$ in cohort 1 patients with chemotherapyresistant disease who received pembrolizumab/BL-8040 with increased $\mathrm{CD}^{+}$effector T-cell tumor infiltration, and decreases in both myeloid-derived suppressor cells and circulating regulatory T-cells (15). The disease control rate was $77 \%$ (median duration of response $=7.8$ months) in cohort 2 patients who received pembrolizumab/BL-8040 in conjunction with combination chemotherapy comprising liposomal irinotecan, 5-fluorouracil and leucovorin (15).

The success of COMBAT along with other immunotherapy approaches currently being tested will expand the armamentarium against pancreatic cancer $(9,15)$. A detailed understanding of the individual patient's response to the different forms of treatment will be necessary to further improve the prognosis of pancreatic cancer patients. The relationship between molecular subtypes (most notably the immunogenic subtype) as described by Bailey et al., and the responsiveness to evolving immunotherapeutic strategies needs to be investigated (10). Single cell and spatial transcriptomic approaches now allow single cell profiling of tumour and immune cell populations resident in patient tumors. These approaches are providing unparalleled insights into immune-tumor interactions and offer new opportunities for targeted immunotherapeutic intervention.

\section{Acknowledgments}

Funding: None.

\section{Footnote}

Provenance and Peer Review: This article was commissioned by the editorial office of Hepatobiliary Surgery and Nutrition. The article did not undergo external peer review.

Conflicts of Interest: All authors have completed the ICMJE uniform disclosure form (available at https://hbsn. amegroups.com/article/view/10.21037/hbsn-21-238/coif). Dr. CS has received payments for lectures and advisory boards from Roche, MSD, Bayer, Servier, Astra Zeneca and Eisai. The other authors have no conflicts of interest to declare.

Ethical Statement: The authors are accountable for all aspects of the work in ensuring that questions related to the accuracy or integrity of any part of the work are appropriately investigated and resolved.

Open Access Statement: This is an Open Access article distributed in accordance with the Creative Commons Attribution-NonCommercial-NoDerivs 4.0 International License (CC BY-NC-ND 4.0), which permits the noncommercial replication and distribution of the article with the strict proviso that no changes or edits are made and the original work is properly cited (including links to both the formal publication through the relevant DOI and the license). See: https://creativecommons.org/licenses/by-nc-nd/4.0/.

\section{References}

1. Klaiber U, Hackert T, Neoptolemos JP. Adjuvant treatment for pancreatic cancer. Transl Gastroenterol Hepatol 2019;4:27.

2. Ghaneh P, Palmer DH, Cicconi S, et al. ESPAC-5F: Fourarm, prospective, multicenter, international randomized phase II trial of immediate surgery compared with neoadjuvant gemcitabine plus capecitabine (GEMCAP) or FOLFIRINOX or chemoradiotherapy (CRT) in patients with borderline resectable pancreatic cancer. J Clin Oncol 2020;38:4505. 
3. Imamura M, Doi R, Imaizumi T, et al. A randomized multicenter trial comparing resection and radiochemotherapy for resectable locally invasive pancreatic cancer. Surgery 2004;136:1003-11.

4. Hackert T, Sachsenmaier M, Hinz U, et al. Locally Advanced Pancreatic Cancer: Neoadjuvant Therapy With Folfirinox Results in Resectability in $60 \%$ of the Patients. Ann Surg 2016;264:457-63.

5. Katz MHG, Shi Q, Meyers JP, et al. Alliance A021501: Preoperative mFOLFIRINOX or mFOLFIRINOX plus hypofractionated radiation therapy (RT) for borderline resectable (BR) adenocarcinoma of the pancreas. J Clin Oncol 2021;39:377.

6. Hewitt DB, Nissen N, Hatoum H, et al. A phase 3 randomized clinical trial of chemotherapy with or without algenpantucel-L (hyperacute-pancreas) immunotherapy in subjects with borderline resectable or locally advanced unresectable pancreatic cancer. Ann Surg 2020. doi: 10.1097/SLA.0000000000004669. [Epub ahead of print].

7. McCormick KA, Coveler AL, Rossi GR, et al. Pancreatic cancer: Update on immunotherapies and algenpantucel-L. Hum Vaccin Immunother 2016;12:563-75.

8. Seifert L, Werba G, Tiwari S, et al. Radiation Therapy Induces Macrophages to Suppress T-Cell Responses Against Pancreatic Tumors in Mice. Gastroenterology 2016;150:1659-72.e5.

Cite this article as: Springfeld C, Bailey $\mathrm{P}$, Hackert T, Neoptolemos JP. Perioperative immunotherapy for pancreatic cancer is on its way. HepatoBiliary Surg Nutr 2021;10(4):534-537. doi: $10.21037 / \mathrm{hbsn}-21-238$
9. Bear AS, Vonderheide RH, O'Hara MH. Challenges and Opportunities for Pancreatic Cancer Immunotherapy. Cancer Cell 2020;38:788-802.

10. Bailey P, Chang DK, Nones K, et al. Genomic analyses identify molecular subtypes of pancreatic cancer. Nature 2016;531:47-52.

11. Balachandran VP, Łuksza M, Zhao JN, et al. Identification of unique neoantigen qualities in long-term survivors of pancreatic cancer. Nature 2017;551:512-6.

12. Moral JA, Leung J, Rojas LA, et al. ILC2s amplify PD-1 blockade by activating tissue-specific cancer immunity. Nature 2020;579:130-5.

13. Candido JB, Morton JP, Bailey P, et al. CSF1R(+) Macrophages Sustain Pancreatic Tumor Growth through T Cell Suppression and Maintenance of Key Gene Programs that Define the Squamous Subtype. Cell Rep 2018;23:1448-60.

14. Steele CW, Karim SA, Leach JDG, et al. CXCR2 Inhibition Profoundly Suppresses Metastases and Augments Immunotherapy in Pancreatic Ductal Adenocarcinoma. Cancer Cell 2016;29:832-45.

15. Bockorny B, Semenisty V, Macarulla T, et al. BL-8040, a CXCR4 antagonist, in combination with pembrolizumab and chemotherapy for pancreatic cancer: the COMBAT trial. Nat Med 2020;26:878-85. 